\title{
Abdominal Infection, CTCAE
}

National Cancer Institute

\section{Source}

National Cancer Institute. Abdominal Infection, CT CAE. NCI Thesaurus. Code C143254.

A disorder characterized by an infectious process involving the abdominal cavity. 